\title{
UNIVERSALITY OF RANK 6 PLÜCKER RELATIONS AND GRASSMANN CONE PRESERVING MAPS
}

\author{
ALEX KASMAN, KATHRYN PEDINGS, AMY REISZL, AND TAKAHIRO SHIOTA
}

(Communicated by Michael Stillman)

\begin{abstract}
The Plücker relations define a projective embedding of the Grassmann variety $\operatorname{Gr}(p, n)$. We give another finite set of quadratic equations which defines the same embedding, and whose elements all have rank 6 . This is achieved by constructing a certain finite set of linear maps $\bigwedge^{p} k^{n} \rightarrow \bigwedge^{2} k^{4}$, and pulling back the unique Plücker relation on $\bigwedge^{2} k^{4}$. We also give a quadratic equation depending on $(p+2)$ parameters having the same properties.
\end{abstract}

\section{INTRODUCTION}

1.1. Plücker relations. Throughout, let $k$ be a field, and let $e_{1}, \ldots, e_{n}$ be a basis of the vector space $k^{n}$. Define the coordinates $\left\{\Pi_{i_{1}, \ldots, i_{p}}\right\}_{1 \leq i_{1}<\cdots<i_{p} \leq n}$ on $\bigwedge^{p} k^{n}$ by

$$
\bigwedge^{p} k^{n} \ni \omega=\sum_{1 \leq i_{1}<i_{2}<\cdots<i_{p} \leq n} \Pi_{i_{1} i_{2} \ldots i_{p}} e_{i_{1}} \wedge e_{i_{2}} \wedge \cdots \wedge e_{i_{p}}
$$

and extend them to arbitrary indices in $\{1, \ldots, n\}^{p}$ by making them antisymmetric.

An element $\omega \in \bigwedge^{p} k^{n}$ is called decomposable if it can be written in the form $\omega=v_{1} \wedge v_{2} \wedge \cdots \wedge v_{p}$ for some $v_{i} \in k^{n}$; otherwise it is called indecomposable. The Grassmann cone $\Gamma^{p} k^{n}=\left\{\omega \in \bigwedge^{p} k^{n} \mid \omega=v_{1} \wedge v_{2} \wedge \cdots \wedge v_{p}\right.$ for some $\left.v_{i} \in k^{n}\right\}$ is the set of decomposable elements in $\bigwedge^{p} k^{n}$. The Plücker relations $[3,7,8,9]$

$$
P_{A, B}(\omega):=\sum_{i=1}^{p+1}(-1)^{i-1} \Pi_{a_{1} a_{2} \ldots a_{p-1} b_{i}} \Pi_{b_{1} b_{2} \ldots b_{p+1} \backslash b_{i}}=0,
$$

where $A=\left\{a_{1}, \ldots, a_{p-1}\right\}, B=\left\{b_{1}, \ldots, b_{p+1}\right\} \subset\{1, \ldots, n\}$, and where the $\backslash b_{i}$ at the end of the indices indicates the absence of $b_{i}$ from the indices, hold if and only if $\omega \in \Gamma^{p} k^{n}$, making $\Gamma^{p} k^{n}$ a $k^{\times}$-invariant affine variety. ${ }^{1}$ The quotient $\operatorname{Gr}(p, n):=$ $\left(\Gamma^{p} k^{n} \backslash\{0\}\right) / k^{\times} \subset \mathbb{P}\left(\bigwedge^{p} k^{n}\right)$ is the Grassmann variety.

We do not need to consider all the choices of indices $A$ and $B$ : Since rearranging the elements of $A$ or $B$ only affects $P_{A, B}$ by total change in sign, it suffices to consider $A$ and $B$ whose elements are listed in increasing order. Moreover, if $A \subset B$,

Received by the editors September 30, 2005 and, in revised form, January 31, 2007.

2000 Mathematics Subject Classification. Primary 14M15, 15A75.

${ }^{1}$ To be precise, while (2) cut out $\Gamma^{p} k^{n}$ set-theoretically for any $k$ [9, Theorem 1], more general Plücker relations may be needed to define it scheme-theoretically if $\operatorname{char}(k)>0[1]$. In this paper we shall concentrate on the set-theoretic aspect, so the simpler Plücker relations as in (2) suffice. 
then $P_{A, B}=0$, and if $A \backslash(A \cap B)$ is a one point set $\{a\}$, then exchanging $a$ with any element of $B \backslash(A \cap B)$ only affects $P_{A, B}$ by total change in sign. So we take

$$
\mathcal{P}(p, n)=\left\{\begin{array}{l|l}
P_{A, B} & \begin{array}{l}
A, B \subset\{1, \ldots, n\}, A=\left\{a_{1}, \ldots, a_{p-1}\right\}, B=\left\{b_{1}, \ldots, b_{p+1}\right\} \\
\text { with } a_{1}<\cdots<a_{p-1} \text { and } b_{1}<\cdots<b_{p+1}, A \not \subset B, \text { and } \\
\text { if } A \backslash(A \cap B)=\{a\} \text { then } a<b \text { for any } b \in B \backslash(A \cap B)
\end{array}
\end{array}\right\}
$$

as a set of generators of Plücker relations, and it suffices to define the Grassmann cone: $\Gamma^{p} k^{n}=\left\{\omega \in \bigwedge^{p} k^{n} \mid P(\omega)=0\right.$ for all $\left.P \in \mathcal{P}(p, n)\right\}$.

By definition we have $\mathcal{P}(p, n)=\emptyset$ if $\min \{p, n-p\} \leq 1$. The first nontrivial case $(p, n)=(2,4)$ yields $\mathcal{P}(2,4)=\left\{P_{\{1\}\{2,3,4\}}\right\}$, so $\omega=\sum_{1 \leq i<j \leq 4} \Pi_{i j} e_{i} \wedge e_{j} \in \bigwedge^{2} k^{4}$ is decomposable if and only if

$$
P_{\{1\}\{2,3,4\}}(\omega)=\Pi_{12} \Pi_{34}-\Pi_{13} \Pi_{24}+\Pi_{14} \Pi_{23}=0 .
$$

The rank of a quadratic form is the rank of the symmetric matrix which defines it (unless $\operatorname{char}(k)=2$, in which case a different definition of rank is used [4]). The rank of $P_{A, B}$ as a quadratic form on $\bigwedge^{p} k^{n}$ is twice the number $|B \backslash(A \cap B)|$ of nonvanishing terms in $(2)$. So the set $\mathcal{P}(p, n)$ consists of quadratic forms of every even rank from 6 up to $2 \min \{p, n-p\}+2$, and the Plücker relations in $\mathcal{P}(p, n)$ all have rank 6 only when $\min \{p, n-p\}=2$. The literature on algebraic geometry occasionally demonstrates an interest in the rank of the Plücker relations $[13,14]$, with particular attention paid to the simplest, rank 6 case.

1.2. Grassmann cone preserving maps. A linear map $G: \bigwedge^{p} k^{n} \rightarrow \bigwedge^{p^{\prime}} k^{n^{\prime}}$ is said to be a Grassmann cone preserving map (GCP map for short) if

$$
G\left(\Gamma^{p} k^{n}\right) \subset \Gamma^{p^{\prime}} k^{n^{\prime}}
$$

The induced map $\bigwedge^{p} L: \bigwedge^{p} k^{n} \rightarrow \bigwedge^{p} k^{n^{\prime}}$ of a linear map $L: k^{n} \rightarrow k^{n^{\prime}}$ is a GCP map. Another GCP map is the dual isomorphism $\delta: \bigwedge^{p} k^{n} \rightarrow \bigwedge^{n-p} k^{n}$, defined by

$$
e_{i_{1}} \wedge \cdots \wedge e_{i_{p}} \mapsto e_{j_{1}} \wedge \cdots \wedge e_{j_{n-p}}
$$

where $i_{1}<\cdots<i_{p}, j_{1}<\cdots<j_{n-p}$ and $\left\{i_{1}, \ldots, i_{p}\right\} \cup\left\{j_{1}, \ldots, j_{n-p}\right\}=\{1, \ldots, n\}$.

Every nontrivial GCP map (those which do not send the whole of $\bigwedge^{p} k^{n}$ to decomposables) can be written as a composition of maps of these two types [18].

1.3. Motivation. In the theory of classical integrable systems, it is well-known that the KP hierarchy of soliton equations, written in an appropriate (Hirota) form, is nothing but the Plücker relations for an infinite dimensional Grassmannian in the space of functions $[2,15]$. Somewhat remarkably, in that setting a single 3 -term (i.e., rank 6) quadratic functional equation with parameters suffices to encode the entire hierarchy $[6,12,17]$, and the same 3 -term equation, even without the parameters, can characterize Jacobians of curves among all the principally polarized abelian varieties [10]. However, the literature on algebraic geometry does not appear to have a similar result on the "universality" of the 3-term Plücker relation (3), i.e., that the 3 -term relation is in a sense the only Plücker relation one needs.

In this paper, we will show such universality by pulling back (3) by various GCP maps to obtain a finite set of rank 6 equations on $\bigwedge^{p} k^{n}$ which suffices to cut out the cone of decomposables $\Gamma^{p} k^{n} \subset \bigwedge^{p} k^{n}$ set-theoretically. Inspired by the soliton theory observation, we will also construct a parameter-dependent rank 6 equation which determines the decomposability of $\omega$. 


\section{A SET OF POLYNOMials With QUADRIC RANK 6}

We will define another set of quadratic forms $\mathcal{P}^{\prime}(p, n)$ to be used later to cut out the Grassmann cone $\Gamma^{p} k^{n}$ just as $\mathcal{P}(p, n)$ does. First we introduce a convenient notation: for a 2-vector $\vec{i}_{j}=\left(i_{j}^{(0)}, i_{j}^{(1)}\right) \in\{1, \ldots, n\}^{2}$, let

$$
\Pi_{i_{1} i_{2} \ldots i_{j-1} \vec{i}_{j} i_{j+1} \ldots i_{p}}=\Pi_{i_{1} i_{2} \ldots i_{j-1} i_{j}^{(0)}} i_{j+1 \ldots i_{p}}+\Pi_{i_{1} i_{2} \ldots i_{j-1} i_{j}^{(1)}} i_{j+1 \ldots i_{p}},
$$

and extend it inductively to the case where two or more indices are 2-vectors.

Definition 2.1. For $\mathcal{A}=\left\{\alpha_{1}, \alpha_{2}, \alpha_{3}, \alpha_{4}\right\} \subset\{1, \ldots, n\}, \mathcal{B}=\left\{\vec{\beta}_{1}, \vec{\beta}_{2}, \ldots, \vec{\beta}_{m}\right\}$ with $\vec{\beta}_{i}=\left(\beta_{i}^{(0)}, \beta_{i}^{(1)}\right) \in\{1, \ldots, n\}^{2}$, and $\mathcal{C}=\left\{\gamma_{1}, \ldots, \gamma_{p-m-2}\right\} \subset\{1, \ldots, n\}$, let

(4) $P_{\mathcal{A}, \mathcal{B}, \mathcal{C}}^{\prime}=\pi_{12} \pi_{34}-\pi_{13} \pi_{24}+\pi_{14} \pi_{23}$, where $\pi_{i j}=\Pi_{\alpha_{i} \alpha_{j} \vec{\beta}_{1} \ldots \vec{\beta}_{m} \gamma_{1} \ldots \gamma_{p-2-m}}$.

Note that $P_{\mathcal{A}, \mathcal{B}, \mathcal{C}}^{\prime}$ is nothing but (3) with the six variables $\Pi_{i j}$ replaced by $\pi_{i j}$. Thus, it has rank at most six as a quadratic form of the original coordinates $\Pi_{i_{1} \ldots i_{p}}$. Moreover, if $\alpha_{i}, \beta_{j}^{(\mu)}$ and $\gamma_{l}$ are all distinct it has rank exactly six.

Lemma 2.2. Each $P_{\mathcal{A}, \mathcal{B}, \mathcal{C}}^{\prime}$ in (4) can be written as a $\mathbb{Z}$-linear combination of elements of $\mathcal{P}(p, n)$ :

$$
P_{\mathcal{A}, \mathcal{B}, \mathcal{C}}^{\prime}=\sum_{\vec{\mu}, \vec{\nu} \in\{0,1\}^{m}} P_{\left\{\beta_{1}^{\left(\mu_{1}\right)} \ldots \beta_{m}^{\left(\mu_{m}\right)} \gamma_{1} \ldots \gamma_{p-m-2} \alpha_{1}\right\}\left\{\alpha_{2} \alpha_{3} \alpha_{4} \beta_{1}^{\left(\nu_{1}\right)} \ldots \beta_{m}^{(\nu m)} \gamma_{1} \ldots \gamma_{p-m-2}\right\}} .
$$

Proof. For each fixed choice of $\vec{\mu}$ and $\vec{\nu}$ the first three terms in expansion (2) of $P$ are exactly those that appear in (4). The next $m$ terms, i.e., the terms involving $\beta_{i}^{\left(\nu_{i}\right)}$, are zero if $\mu_{i}=\nu_{i}$, and cancel if $\mu_{i} \neq \nu_{i}$ with the term where $\mu_{i}$ and $\nu_{i}$ are switched. The last $p-m-2$ terms, the terms involving $\gamma_{i}$, are all zero.

The lemma implies that if some $P_{\mathcal{A}, \mathcal{B}, \mathcal{C}}^{\prime}(\omega) \neq 0$, then $\omega$ is indecomposable. Conversely, in Section 4 we will see that some $P_{\mathcal{A}, \mathcal{B}, \mathcal{C}}^{\prime}(\omega) \neq 0$ if $\omega$ is indecomposable. However, not every element of $\mathcal{P}(p, n)$ is a linear combination of rank 6 quadratic forms $P_{\mathcal{A}, \mathcal{B}, \mathcal{C}}^{\prime}$. For instance, a tedious but straightforward computation shows that the Plücker relation $P_{\{1,2,3\},\{4,5,6,7,8\}}$ cannot be a linear combination of $P_{\mathcal{A}, \mathcal{B}, \mathcal{C}}^{\prime}$.

The quadratic forms $P_{\mathcal{A}, \mathcal{B}, \mathcal{C}}^{\prime}$ are not independent. Clearly, rearranging the elements of each index set affects them by at most a change in sign. If the indices $\alpha_{i}$, $\beta_{j}^{(\varepsilon)}$ and $\gamma_{l}$ are not all distinct, then $P_{\mathcal{A}, \mathcal{B}, \mathcal{C}}^{\prime}$ is a linear combination of those with distinct indices. There are other, less obvious linear relations like

$$
\sum_{i \in \mathbb{Z} / 3 \mathbb{Z}} P_{\left\{\nu_{i}, \alpha_{1}, \alpha_{2}, \alpha_{3}\right\}, \mathcal{B} \cup\left\{\left(\nu_{i+1}, \nu_{i+2}\right)\right\}, \mathcal{C}}^{\prime}-\sum_{0 \leq i, j \leq 2 ; i \neq j} P_{\left\{\nu_{i}, \alpha_{1}, \alpha_{2}, \alpha_{3}\right\}, \mathcal{B}, \mathcal{C} \cup\left\{\nu_{j}\right\}}^{\prime}=0 .
$$

However, instead of using these identities to find a basis for the linear span of the set of all $P_{\mathcal{A}, \mathcal{B}, \mathcal{C}}^{\prime}$, let us introduce a subset just suited for our purpose of set-theoretic characterization of the Grassmann cone.

Definition 2.3. Let $\mathcal{P}^{\prime}(p, n)$ be the set of all $P_{\mathcal{A}, \mathcal{B}, \mathcal{C}}^{\prime}$ where the triples $\mathcal{A}, \mathcal{B}, \mathcal{C}$ satisfy

(5) $\left\{\begin{array}{l}\text { the indices } \alpha_{i}, \beta_{j}^{(\mu)} \text { and } \gamma_{l} \text { are all distinct and such that } \\ \alpha_{i}<\alpha_{i+1}, \quad \beta_{i}^{(0)}<\beta_{i}^{(1)}, \quad \beta_{i}^{(\nu)}<\beta_{i+1}^{(\nu)}, \quad \beta_{i}^{(0)}<\alpha_{1}, \quad \beta_{i}^{(1)}<\alpha_{3}, \quad \gamma_{i}<\gamma_{i+1} .\end{array}\right.$ 
It is a simple exercise in combinatorics to find the number of elements of $\mathcal{P}^{\prime}(p, n)$ :

$$
\left|\mathcal{P}^{\prime}(p, n)\right|=\sum_{m=0}^{M} \frac{n !}{(2 m+4) !(p-m-2) !(n-p-m-2) !}\left(C_{m+2}-C_{m+1}\right),
$$

where $M=\min \{p, n-p\}-2$, and $C_{r}=\frac{1}{r+1}\left(\begin{array}{c}2 r \\ r\end{array}\right)$ is the Catalan number [16]. Rewriting this, we see that in general $\mathcal{P}^{\prime}(p, n)$ is a much smaller set than $\mathcal{P}(p, n)$ :

$$
\left|\mathcal{P}^{\prime}(p, n)\right|=\sum_{m=0}^{M} \frac{3 m+3}{(2 m+4)(2 m+3)} a_{m} \leq \frac{1}{4} a_{0}+\sum_{m=1}^{M} a_{m}=|\mathcal{P}(p, n)|,
$$

where the equality holds if and only if $M \leq 0$, and where

$$
a_{m}:=\frac{n !}{(m+1) !(m+3) !(p-m-2) !(n-p-m-2) !}
$$

is the number of pairs of subsets $A, B \subset\{1, \ldots, n\}$ such that $|A|=p-1,|B|=p+1$ and $|A \cap B|=p-m-2$, which is also the number of the rank $2 m+6$ elements in $\mathcal{P}(p, n)$ if $m>0$, and is four times this number if $m=0$.

\section{Decomposability And GCP maps to $\Gamma^{2} k^{4}$}

In this section we will construct a finite set of GCP maps from $\bigwedge^{p} k^{n}$ to $\bigwedge^{2} k^{4}$, indexed by the same triples $\mathcal{A}, \mathcal{B}, \mathcal{C}$ as in Definition 2.3, such that if $\omega$ is indecomposable, then for some GCP map $G$ in this set $G(\omega)$ is also indecomposable. First we define a linear map from $k^{n}$ to $k^{p+2}$ determined by these indices and prove a lemma addressing a question of vector geometry.

For any $S \subset\{1, \ldots, n\}$ let $k^{S}=\bigoplus_{i \in S} k e_{i} \subset k^{n}$. For any $S, T \subset\{1, \ldots, n\}$ and a map $f: S \rightarrow T$, define $f^{*}: k^{T} \rightarrow k^{S}$ and $f_{*}: k^{S} \rightarrow k^{T}$ by $\sum_{j \in T} a_{j} e_{j} \mapsto \sum_{i \in S} a_{f(i)} e_{i}$ and $\sum_{i \in S} a_{i} e_{i} \mapsto \sum_{i \in S} a_{i} e_{f(i)}$, respectively. Thus if $i_{S}$ is the natural inclusion $S \subset\{1, \ldots, n\}$, then $\pi_{S}:=i_{S}^{*}: k^{n} \rightarrow k^{S}$ is the projection $\sum_{i=1}^{n} a_{i} e_{i} \mapsto \sum_{i \in S} a_{i} e_{i}$. Writing $S=\left\{\xi_{1}, \ldots, \xi_{|S|}\right\}$ with $\xi_{1}<\cdots<\xi_{|S|}$, we also define $\tau_{S}: S \rightarrow\{1, \ldots,|S|\}$ by $\tau_{S}\left(\xi_{i}\right)=i$, so that $\tau_{S *}: k^{S} \stackrel{\sim}{\rightarrow} k^{|S|}, \tau_{S *}\left(e_{\xi_{i}}\right)=e_{i}$, is an isomorphism.

Definition 3.1. Suppose $\mathcal{A}, \mathcal{B}$ and $\mathcal{C}$ are as in Definition 2.1, subject to condition (5). Let $B_{\varepsilon}=\left\{\beta_{1}^{(\varepsilon)}, \ldots, \beta_{m}^{(\varepsilon)}\right\}(\varepsilon=0,1)$, and let $S^{\prime}=\mathcal{A} \cup B_{1} \cup \mathcal{C}$ and $S=S^{\prime} \cup B_{0}$. Let $\varphi: B_{0} \stackrel{\sim}{\rightarrow} B_{1}$ be the order-preserving map $\varphi\left(\beta_{i}^{(0)}\right)=\beta_{i}^{(1)}, i=1, \ldots, m$, and extend it to a map from $S$ to $S^{\prime}$, still denoted by $\varphi$, by $\varphi(i)=i$ outside $B_{0}$. Noting that $\left|S^{\prime}\right|=p+2$, we let $X:=X_{\mathcal{A}, \mathcal{B}, \mathcal{C}}: k^{n} \rightarrow k^{p+2}$ be the composition of linear maps $\tau_{S^{\prime} *} \circ \varphi_{*} \circ \pi_{S}: k^{n} \rightarrow k^{S} \rightarrow k^{S^{\prime}} \rightarrow k^{p+2}$, i.e., writing $S^{\prime}=\left\{\xi_{1}, \ldots, \xi_{\left|S^{\prime}\right|}\right\}$ with $\xi_{1}<\cdots<\xi_{\left|S^{\prime}\right|}$ we have

$$
X\left(e_{i}\right)= \begin{cases}e_{j} & \text { if } i=\xi_{j}, \text { or if } \exists l\left[i=\beta_{l}^{(0)} \text { and } \beta_{l}^{(1)}=\xi_{j}\right], \\ 0 & \text { otherwise. }\end{cases}
$$

Lemma 3.2. Let $V_{0}$ and $V_{1}$ be $p$-dimensional subspaces of $k^{n}$. If $q:=q\left(V_{0}, V_{1}\right):=$ $p-\operatorname{dim}\left(V_{0} \cap V_{1}\right)=\operatorname{dim}\left(V_{0}+V_{1}\right)-p$ satisfies $q \geq 2$, there exist $\mathcal{A}, \mathcal{B}$ and $\mathcal{C}$ subject to (5) so that

- $\hat{V}_{0}=X_{\mathcal{A}, \mathcal{B}, \mathcal{C}}\left(V_{0}\right)$ and $\hat{V}_{1}=X_{\mathcal{A}, \mathcal{B}, \mathcal{C}}\left(V_{1}\right)$ are $p$-dimensional subspaces of $k^{p+2}$,

- $\operatorname{dim}\left(\hat{V}_{0} \cap \hat{V}_{1}\right)=p-2$,

- $\left\{X_{\mathcal{A}, \mathcal{B}, \mathcal{C}}\left(e_{\alpha_{i}}\right) \mid i=1,2,3,4\right\}$ is linearly independent modulo $\left(\hat{V}_{0} \cap \hat{V}_{1}\right)$. 
Proof. Take a minimal $S \subset\{1, \ldots, n\}$ such that $\left.\pi_{S}\right|_{V_{\varepsilon}}: V_{\varepsilon} \rightarrow k^{S}(\varepsilon=0,1)$ are injective, and such that $q_{S}:=q\left(\pi_{S}\left(V_{0}\right), \pi_{S}\left(V_{1}\right)\right) \geq 2$. Let $V_{\varepsilon}^{\prime}:=\pi_{S}\left(V_{\varepsilon}\right)$. We have

$$
q_{S} \leq q
$$

since $V_{0}^{\prime} \cap V_{1}^{\prime} \supset \pi_{S}\left(V_{0} \cap V_{1}\right) \simeq V_{0} \cap V_{1}$ implies $p-q_{S} \geq p-q$, and

$$
p+q_{S}=|S|
$$

since the minimality of $S$ implies $V_{0}^{\prime}+V_{1}^{\prime}=\pi_{S}\left(V_{0}+V_{1}\right)=k^{S}$.

If $q_{S}=2$ so that $\operatorname{dim} k^{S} /\left(V_{0}^{\prime} \cap V_{1}^{\prime}\right)=4$, choose $\mathcal{A} \subset S$ such that $|\mathcal{A}|=4$ and the composition of the maps $k^{\mathcal{A}} \hookrightarrow k^{S} \rightarrow k^{S} /\left(V_{0}^{\prime} \cap V_{1}^{\prime}\right)$ is an isomorphism, and let $\mathcal{B}=\emptyset$ and $\mathcal{C}=S \backslash \mathcal{A}$. It is easy to see that $X_{\mathcal{A}, \mathcal{B}, \mathcal{C}}$ satisfies the desired properties.

If $q_{S}>2$, then for any $i \in S$ the minimality of $S$ implies that $\left.\pi_{S \backslash\{i\}}\right|_{V_{0}}$ or $\left.\pi_{S \backslash\{i\}}\right|_{V_{1}}$ cannot be injective (otherwise we would have $q_{S \backslash\{i\}}=q_{S}-1 \geq 2$ ), thus

$$
e_{i} \in V_{0}^{\prime} \quad \text { or } \quad e_{i} \in V_{1}^{\prime} \quad(\forall i \in S) .
$$

Take any $B_{0}, B_{1} \subset S$ such that

$$
k^{B_{\varepsilon}} \subset V_{\varepsilon}^{\prime} \quad \text { and } \quad k^{B_{\varepsilon}} \cap V_{1-\varepsilon}^{\prime}=\{0\} \quad(\varepsilon=0,1) .
$$

Note that (8) implies $B_{0} \cap B_{1}=\emptyset$ and $\left|B_{\varepsilon}\right| \leq q_{S}$. Let $\delta=0$ if $\left|B_{0}\right| \leq\left|B_{1}\right|$; otherwise let $\delta=1$. Let $S^{\prime}=S \backslash B_{\delta}$. Take any inclusion $\varphi: B_{\delta} \hookrightarrow B_{1-\delta} \subset S^{\prime}$, and extend it to a map from $S$ to $S^{\prime}$ by letting $\varphi(i)=i$ for $i \in S^{\prime}$. As a consequence of (8) we have $\operatorname{ker} \varphi_{*} \cap V_{\varepsilon}^{\prime}=\{0\}$, so that $V_{\varepsilon}^{\prime \prime}:=\varphi_{*}\left(V_{\varepsilon}^{\prime}\right)$ are $p$-dimensional subspaces of $k^{S^{\prime}}$. Since $V_{0}^{\prime \prime}+V_{1}^{\prime \prime}=\varphi_{*}\left(V_{0}^{\prime}+V_{1}^{\prime}\right)=\varphi_{*}\left(k^{S}\right)=k^{S^{\prime}}$ has dimension $|S|-\left|B_{\delta}\right|=p+q_{S}-\left|B_{\delta}\right|$ (see (6)), we have

$$
q\left(V_{0}^{\prime \prime}, V_{1}^{\prime \prime}\right)=q_{S}-\left|B_{\delta}\right|=q_{S}-\min \left\{\left|B_{0}\right|,\left|B_{1}\right|\right\} .
$$

If we further assume that $\left(B_{0}, B_{1}\right)$ is a maximal pair satisfying (8), then $\left|B_{0}\right|=$ $\left|B_{1}\right|=q_{S}$. To prove this, it suffices to show that $B_{\delta}$ is not maximal if $\left|B_{\delta}\right|<q_{S}$. By (7) we have

$$
e_{i} \in V_{0}^{\prime \prime} \quad \text { or } \quad e_{i} \in V_{1}^{\prime \prime} \quad\left(\forall i \in S^{\prime}\right),
$$

but since $\left|\left\{i \in S^{\prime} \mid e_{i} \in V_{\varepsilon}^{\prime \prime}\right\}\right| \leq \operatorname{dim}\left(V_{\varepsilon}^{\prime \prime}\right)=p(\varepsilon=0,1)$ and $\left|S^{\prime}\right|=p+q_{S}-\left|B_{\delta}\right|$, if $\left|B_{\delta}\right|<q_{S}$, then there exists $i \in S^{\prime}$ such that

$$
e_{i} \notin V_{1-\delta}^{\prime \prime} \text {. }
$$

Let $B_{\delta}^{\prime}=B_{\delta} \cup\{i\}$. Since $i \in S^{\prime}=S \backslash B_{\delta}$ we have $i \notin B_{\delta}$, so that $B_{\delta}^{\prime} \supsetneq B_{\delta}$. By (10) we have $e_{i} \notin V_{1-\delta}^{\prime}$; hence by (7) we have $e_{i} \in V_{\delta}^{\prime}$. This and the first formula in (8) (with $\varepsilon=\delta$ ) yield

$$
k^{B_{\delta}^{\prime}}=k^{B_{\delta}} \oplus k e_{i} \subset V_{\delta}^{\prime} .
$$

Using (10), the first formula in (8) with $\varepsilon=1-\delta$ and the second formula in (8) with $\varepsilon=\delta$, we have

$$
e_{i} \notin \varphi_{*}^{-1}\left(V_{1-\delta}^{\prime \prime}\right)=V_{1-\delta}^{\prime} \oplus \bigoplus_{i \in B_{\delta}} k\left(e_{i}-e_{\varphi(i)}\right)=V_{1-\delta}^{\prime} \oplus k^{B_{\delta}},
$$

hence $k^{B_{\delta}^{\prime}} \cap V_{1-\delta}^{\prime}=\{0\}$, which together with (11) shows that $B_{\delta}$ is not maximal.

Take maximal $B_{\varepsilon}$, denote them by $\bar{B}_{\varepsilon}$, and write $\bar{B}_{\varepsilon}=\left\{b_{1}^{\varepsilon}, \ldots, b_{q_{S}}^{\varepsilon}\right\}$ with $b_{1}^{\varepsilon}<$ $\cdots<b_{q_{S}}^{\varepsilon}$. We then let $B_{\varepsilon}=\left\{b_{1}^{\varepsilon}, \ldots, b_{q_{S}-2}^{\varepsilon}\right\} \subset \bar{B}_{\varepsilon}$,

$$
\begin{gathered}
\mathcal{A}=\left\{b_{q_{S}-1}^{0}, b_{q_{S}}^{0}, b_{q_{S}-1}^{1}, b_{q_{S}}^{1}\right\}=\left(\bar{B}_{0} \backslash B_{0}\right) \cup\left(\bar{B}_{1} \backslash B_{1}\right), \\
\mathcal{B}=\left\{\left(b_{i}^{0}, b_{i}^{1}\right) \mid i=1, \ldots, q_{S}-2\right\} \quad \text { and } \quad \mathcal{C}=S \backslash\left(\bar{B}_{0} \cup \bar{B}_{1}\right)=S \backslash\left(\mathcal{A} \cup B_{0} \cup B_{1}\right) .
\end{gathered}
$$


As subsets of $\bar{B}_{\varepsilon}, B_{\varepsilon}$ satisfy (8). Taking order-preserving $\varphi: B_{0} \stackrel{\sim}{\rightarrow} B_{1}$ in the above argument (note $\delta=0$ since $\left|B_{0}\right|=\left|B_{1}\right|$ ) we obtain $\varphi: S \rightarrow S^{\prime}$ and $X:=$ $X_{\mathcal{A}, \mathcal{B}, \mathcal{C}}=\tau_{S^{\prime} *} \circ \varphi_{*} \circ \pi_{S}$ as in Definition 3.1. The map $X$ is regular on each $V_{\varepsilon}$ (so that $\hat{V}_{\varepsilon}=\tau_{S^{\prime} *} V_{\varepsilon}^{\prime \prime}$ has dimension $p$ ), and $X\left(e_{\alpha_{i}}\right)=\tau_{S^{\prime *}}\left(e_{\alpha_{i}}\right)$ remain linearly independent modulo $\hat{V}_{0} \cap \hat{V}_{1}=\tau_{S^{\prime *}}\left(V_{0}^{\prime \prime} \cap V_{1}^{\prime \prime}\right)$. Finally, applying (9) to $B_{\varepsilon}$ we have $q\left(V_{0}^{\prime \prime}, V_{1}^{\prime \prime}\right)=q_{S}-\left|B_{\delta}\right|=2$, and $\operatorname{dim}\left(\hat{V}_{0} \cap \hat{V}_{1}\right)=\operatorname{dim}\left(V_{0}^{\prime \prime} \cap V_{1}^{\prime \prime}\right)=p-2$ as desired.

Now, let us define a GCP map associated to the same indices:

Definition 3.3. The Grassmann cone preserving map $G:=G_{\mathcal{A}, \mathcal{B}, \mathcal{C}}: \bigwedge^{p} k^{n} \rightarrow$ $\bigwedge^{2} k^{4}$ is defined as

$$
G_{\mathcal{A}, \mathcal{B}, \mathcal{C}}=\bigwedge^{2} Z \circ \delta \circ \bigwedge^{p} X_{\mathcal{A}, \mathcal{B}, \mathcal{C}}
$$

where $X_{\mathcal{A}, \mathcal{B}, \mathcal{C}}$ is as in Definition 3.1, $\delta: \bigwedge^{p} k^{p+2} \rightarrow \bigwedge^{2} k^{p+2}$ is the dual isomorphism, and $Z: k^{p+2} \rightarrow k^{4}$ is the linear map

$$
Z\left(e_{i}\right)= \begin{cases}e_{j} & \text { if } e_{i}=X_{\mathcal{A}, \mathcal{B}, \mathcal{C}}\left(e_{\alpha_{j}}\right) \\ 0 & \text { otherwise }\end{cases}
$$

Theorem 3.4. The element $\omega \in \bigwedge^{p} k^{n}$ is indecomposable if and only if there exists a choice of indices $\mathcal{A}, \mathcal{B}, \mathcal{C}$ so that $G_{\mathcal{A}, \mathcal{B}, \mathcal{C}}(\omega) \in \bigwedge^{2} k^{4}$ is indecomposable.

Proof. Since $G_{\mathcal{A}, \mathcal{B}, \mathcal{C}}$ is a GCP map, if $\omega$ is decomposable $G_{\mathcal{A}, \mathcal{B}, \mathcal{C}}(\omega)$ is also decomposable for any choice of $\mathcal{A}, \mathcal{B}$, and $\mathcal{C}$. So, let us assume that $\omega$ is indecomposable and show that for an appropriate choice of the indices, its image in $\bigwedge^{2} k^{4}$ is also indecomposable.

Suppose first that $\omega$ can be written as a sum of two decomposable elements

$$
\omega=v_{1} \wedge \cdots \wedge v_{p}+w_{1} \wedge \cdots \wedge w_{p}
$$

Then $\omega$ is indecomposable if and only if [18] the $p$-dimensional subspaces $V_{0}=$ $\left\langle v_{1}, \ldots, v_{p}\right\rangle$ and $V_{1}=\left\langle w_{1}, \ldots, w_{p}\right\rangle$ of $k^{n}$ satisfy $\operatorname{dim}\left(V_{0} \cap V_{1}\right) \leq p-2$. Applying Lemma 3.2 gives us a choice of $\mathcal{A}, \mathcal{B}, \mathcal{C}$ such that

$$
\left(\bigwedge^{p} X_{\mathcal{A}, \mathcal{B}, \mathcal{C}}\right)(\omega)=\omega_{1} \wedge \omega_{2} \in \bigwedge^{p} k^{p+2},
$$

where $\omega_{1}$ is an indecomposable element of the second exterior power of the 4dimensional space $k^{A^{\prime}}:=X_{\mathcal{A}, \mathcal{B}, \mathcal{C}}\left(k^{\mathcal{A}}\right)$, and $\omega_{2}$ is a nonzero element of $\bigwedge^{p-2}\left(\hat{V}_{0} \cap \hat{V}_{1}\right)$. Since $k^{A^{\prime}} \cap\left(\hat{V}_{0} \cap \hat{V}_{1}\right)=\{0\}$, letting $A^{\prime \prime}:=\{1, \ldots, p+2\} \backslash A^{\prime}$ we have $\omega_{2,0}:=$ $\left(\bigwedge^{p-2} \pi_{A^{\prime \prime}}\right) \omega_{2} \neq 0$, which implies $\bigwedge^{2} \pi_{A^{\prime}} \circ \delta\left(\pi_{1} \wedge \pi_{2}\right)=\bigwedge^{2} \pi_{A^{\prime}} \circ \delta\left(\pi_{1} \wedge \pi_{2,0}\right)=c \delta_{A^{\prime}}\left(\pi_{1}\right)$, where $c \neq 0$ and $\delta_{A^{\prime}}$ is the dual isomorphism on $\Lambda^{*} k^{A^{\prime}}$. Since $Z=\tau_{A^{\prime} *} \circ \pi_{A^{\prime}}$, this implies $G_{\mathcal{A}, \mathcal{B}, \mathcal{C}}(\omega)=\bigwedge^{2} Z \circ \delta\left(\omega_{1} \wedge \omega_{2}\right)=\left(\bigwedge^{2} \tau_{A^{\prime *}}\right)\left(c \delta_{A^{\prime}}\left(\omega_{1}\right)\right) \neq 0$.

This proves the claim when $\omega$ can be written as a sum of two decomposable elements. This is always true in $\bigwedge^{2} k^{4}$, but not in general. We proceed by induction on the dimension $n$ with the case $n=4$ as our initialization.

Regarding $k^{n-1}$ as the subspace $\left\langle e_{1}, \ldots, e_{n-1}\right\rangle$ of $k^{n}=\left\langle e_{1}, \ldots, e_{n}\right\rangle$, let $\omega_{1} \in$ $\bigwedge^{p-1} k^{n-1}$ and $\omega_{2} \in \bigwedge^{p} k^{n-1}$ be such that

$$
\omega=\omega_{1} \wedge e_{n}+\omega_{2} .
$$

Now, if $\omega_{1}$ is indecomposable, we make use of the induction hypothesis on $\bigwedge^{p-1} k^{n-1}$ to get $\mathcal{A}, \mathcal{B}$, and $\mathcal{C}$ so that $G_{\mathcal{A}, \mathcal{B}, \mathcal{C}}\left(\omega_{1}\right)$ is indecomposable. If we consider instead 
$\mathcal{C}^{\prime}=\mathcal{C} \cup\{n\}$ which has cardinality one greater than $\mathcal{C}$, then $G_{\mathcal{A}, \mathcal{B}, \mathcal{C}^{\prime}}\left(\omega_{1} \wedge e_{n}\right)=$ $G_{\mathcal{A}, \mathcal{B}, \mathcal{C}}\left(\omega_{1}\right)$ and $G_{\mathcal{A}, \mathcal{B}, \mathcal{C}^{\prime}}\left(\omega_{2}\right)=0$ since $\bigwedge X\left(\omega_{2}\right)=0$. Consequently, $\mathcal{A}, \mathcal{B}, \mathcal{C}^{\prime}$ satisfies the claim.

On the other hand, if $\omega_{2}$ is indecomposable we make use of the induction hypothesis to obtain $\mathcal{A}, \mathcal{B}$ and $\mathcal{C}$. In this case, without any further modification we have that $G(\omega)=G\left(\omega_{2}\right)$ is indecomposable since the absence of the number $n$ in the indices will result in $\bigwedge X$ which annihilates $\omega_{1} \wedge e_{n}$.

The only other possibility is that both $\omega_{1}$ and $\omega_{2}$ are decomposable, which returns us to the case that was proved initially.

\section{Determining Decomposability using the elements of $\mathcal{P}^{\prime}(p, n)$}

Applying Theorem 3.4, we now show that the rank 6 quadratic forms in $\mathcal{P}^{\prime}(p, n)$ are capable of characterizing decomposables, like the Plücker relations in $\mathcal{P}(p, n)$.

Theorem 4.1. For any $\omega \in \bigwedge^{p} k^{n}$, all the elements of $\mathcal{P}^{\prime}(p, n)$ vanish at $\omega$ if and only if $\omega$ is decomposable.

Proof. By Theorem 3.4, $\omega$ is decomposable if and only if $G_{\mathcal{A}, \mathcal{B}, \mathcal{C}}(\omega) \in \bigwedge^{2} k^{4}$ is decomposable for all choices of $\mathcal{A}, \mathcal{B}, \mathcal{C}$ as in Definition 2.3 and $G_{\mathcal{A}, \mathcal{B}, \mathcal{C}}$ as in Definition 3.3. However, the decomposability of an element of $\bigwedge^{2} k^{4}$ is determined by the single Plücker relation (3).

So it is sufficient to note that substituting the 6 coordinates of $G_{\mathcal{A}, \mathcal{B}, \mathcal{C}}(\omega)$ into (3) yields precisely the equation $P_{\mathcal{A}, \mathcal{B}, \mathcal{C}}^{\prime}=0$. To see this, note that writing

$$
G_{\mathcal{A}, \mathcal{B}, \mathcal{C}}(\omega)=\sum_{1 \leq i_{1}<i_{2} \leq 4} \hat{\Pi}_{i_{1} i_{2}} e_{i_{1}} \wedge e_{i_{2}}
$$

we can compute $\hat{\Pi}_{i_{1} i_{2}}$ directly. It is necessarily independent of $\Pi_{j_{1} j_{2} j_{3} \ldots j_{p}}$ unless exactly two of $\alpha_{1}, \ldots, \alpha_{4}$, one each of $\beta_{j}^{(0)}$ and $\beta_{j}^{(1)}$ for each $1 \leq j \leq m$ and all of $\gamma_{1}, \ldots, \gamma_{p-m-2}$ are represented amongst $j_{1}, \ldots, j_{p}$, since the wedge products of which these are the coefficients are in the kernel of $G$. Moreover, of the remaining elements the only restriction on those which arise in $\hat{\Pi}_{i_{1} i_{2}}$ is that $\alpha_{i_{1}}$ and $\alpha_{i_{2}}$ are not present amongst the indices. In particular,

$$
\hat{\Pi}_{i_{1} i_{2}}=\left(\epsilon_{i j}\right) \Pi_{\alpha_{1} \alpha_{2} \alpha_{3} \alpha_{4} \vec{\beta}_{1} \ldots \vec{\beta}_{m} \gamma_{1} \ldots \gamma_{p-2-m} \backslash \alpha_{i_{1}} \alpha_{i_{2}}},
$$

where $\epsilon_{i j} \in\{1,-1\}$ and substituting these into (3) results in $\pm P_{\mathcal{A}, \mathcal{B}, \mathcal{C}}^{\prime}$.

\section{Parameter Dependent Formulation}

In this section we will introduce a GCP map depending polynomially on the $p+2$ free parameters $x_{1}, \ldots, x_{p+2}$, and use it to determine the decomposability of $\omega \in \bigwedge^{p} k^{n}$. Let $R=k\left[x_{1}, \ldots, x_{p+2}\right]$, and let $Q$ be the field of fractions of $R$. We call $\eta \in \bigwedge^{a} R^{b}$ decomposable if $\eta \otimes_{R} Q \in \bigwedge^{a} Q^{b}$ is decomposable, i.e., if $\eta \otimes_{R} Q \in \Gamma^{a} Q^{b}$. If $\eta$ is decomposable, then for any $\mathfrak{p} \in \operatorname{Spec} R$ there exist $v_{1}, \ldots, v_{a} \in\left(R_{\mathfrak{p}}\right)^{b}$ such that $\eta=v_{1} \wedge \cdots \wedge v_{a}$. Here $R_{\mathfrak{p}}$ is the localization of $R$ at $\mathfrak{p}$.

Definition 5.1. Let $X$ be the $(p+2) \times n$ matrix whose $(i, j)$ entry is $x_{i}^{j-1}$, regarded as a $Q$-linear map $X: Q^{n} \rightarrow Q^{p+2}$. Let $Z=(I O)$ be the $4 \times(p+2)$ matrix consisting of a $4 \times 4$ unit matrix and a $4 \times(p-2)$ zero matrix, also regarded as a $Q$-linear map $Z: Q^{p+2} \rightarrow Q^{4}$. The GCP map $\bigwedge^{2} Z \circ \delta \circ \bigwedge^{p} X: \bigwedge^{p} Q^{n} \rightarrow \bigwedge^{2} Q^{4}$ restricted to 
$\bigwedge^{p} k^{n} \subset \bigwedge^{p} Q^{n}$ defines $G: \bigwedge^{p} k^{n} \rightarrow \bigwedge^{2} R^{4}$. Let $H=G^{*} P_{\{1\}\{2,3,4\}}: \bigwedge^{p} k^{n} \rightarrow R$, i.e., for $\omega \in \bigwedge^{p} k^{n}$, writing

$$
G(\omega)=\sum_{1 \leq i<j \leq 4} \hat{\Pi}_{i j} e_{i} \wedge e_{j}, \quad \hat{\Pi}_{i j} \in R
$$

we let

$$
H(\omega)=\hat{\Pi}_{12} \hat{\Pi}_{34}-\hat{\Pi}_{13} \hat{\Pi}_{24}+\hat{\Pi}_{14} \hat{\Pi}_{23} \in R .
$$

Lemma 5.2. For $0 \leq q \leq n$, let $V^{\prime}$ be a q-dimensional subspace of $k^{n}$. Then the $Q$-linear map $X$ restricted to $V^{\prime} \otimes_{k} Q$ has the maximal rank $r:=\min \{p+2, q\}$, i.e., one of the $r \times r$-minors of matrix $X$ is a nonzero element of $R$.

Proof. For any matrix $M$, denote by $M_{i_{1} \ldots i_{a}}^{j_{1} \ldots j_{b}}$ the $a \times b$ matrix obtained by taking the rows $i_{1}, \ldots, i_{a}$ and columns $j_{1}, \ldots, j_{b}$ of $M$. Let $U=\left(u_{i j}\right)$ be an $n \times q$ matrix whose columns form a basis of $V^{\prime}$. It suffices to show that $D:=\operatorname{det}\left((X U)_{1 \ldots r}^{1 \ldots r}\right)$ is not the zero polynomial. Using the Cauchy-Binet formula we have

$$
D=\sum_{n \geq h_{1}>\cdots>h_{r} \geq 1} \operatorname{det} X_{1 \ldots r}^{h_{r} \ldots h_{1}} \operatorname{det} U_{h_{r} \ldots h_{1}}^{1 \ldots r}=\Delta^{\prime} \sum_{n-r \geq \lambda_{1} \geq \cdots \geq \lambda_{r} \geq 0} c_{\lambda} s_{\lambda}\left(x_{1}, \ldots, x_{r}\right),
$$

where $\Delta^{\prime}$ is $(-1)^{r(r-1) / 2}$ times the Vandermonde determinant $\prod_{1<i<j \leq r}\left(x_{i}-x_{j}\right)$, $s_{\lambda}=s_{\lambda}\left(x_{1}, \ldots, x_{r}\right)$ is the Schur function for the partition $\lambda=\left(\lambda_{1}, \bar{\lambda}_{2}, \ldots, \lambda_{r}\right),[11]$, and $c_{\lambda}=\operatorname{det} U_{h_{r} \ldots h_{1}}^{1 \ldots r}$ with $\lambda_{i}=h_{i}-(r-i+1)$. Since the $s_{\lambda}$ are linearly independent over $k$, and since some $c_{\lambda} \in k$ do not vanish, $D$ is not the zero polynomial.

Theorem 5.3. The following four conditions for $\omega \in \bigwedge^{p} k^{n}$ are equivalent:

(1) $\omega$ is decomposable;

(2) $\bigwedge^{p} X(\omega) \in \bigwedge^{p} R^{p+2}$ is decomposable;

(3) $G(\omega) \in \bigwedge^{2} R^{4}$ is decomposable;

(4) $H(\omega)=0$.

Proof. Since $H$ is the pull-back by $G$ of the Plücker relation for $\Gamma^{2} k^{4}$, conditions 3 and 4 are equivalent. Since $G$ is a GCP map, 1 implies 3 . So we have only to prove that 3 implies 2 and 2 implies 1 .

We first prove that 3 implies 2. Suppose $\alpha:=\bigwedge^{p} X(\omega)$ is indecomposable. Then $\beta:=\delta(\alpha) \in \bigwedge^{2} k^{p+2}$ is also indecomposable since $\delta(\beta)=\alpha$ and $\delta$ is a GCP map. Hence for some $P_{A, B} \in \mathcal{P}(2, p+2)$ we have

$$
P_{A, B}(\beta)=\Pi_{i_{0} i_{1}} \Pi_{i_{2} i_{3}}-\Pi_{i_{0} i_{2}} \Pi_{i_{1} i_{3}}+\Pi_{i_{0} i_{3}} \Pi_{i_{1} i_{2}} \neq 0,
$$

where $A=\left\{i_{0}\right\}$ and $B=\left\{i_{1}, i_{2}, i_{3}\right\}$ with $1 \leq i_{1}<\cdots<i_{4} \leq p+2$, and $\beta=$ $\sum_{1 \leq i<j \leq p+2} \Pi_{i j} e_{i} \wedge e_{j}$. Permutation of the parameters $\left(x_{i}\right) \mapsto\left(x_{\sigma(i)}\right)$, where $\sigma \in$ $\mathfrak{S}(p+2)$, does not affect the indecomposability of $\alpha$ and $\beta$, and it yields the action $\Pi_{i j} \mapsto \varepsilon(\sigma, i, j) \Pi_{\sigma(i) \sigma(j)}$ on the coordinates $\Pi_{i j}$ of $\beta$. Here $\varepsilon(\sigma, i, j)= \pm 1$ depends on $i$ and $j$, but in such a way that its net effect on each term of (13) is the same for all three terms. Hence for all choices of $\left(i_{0}, \ldots, i_{3}\right), P_{A, B}(\beta)$ are the same polynomials upon renaming the variables $x_{i}$ and a possible change in sign. In particular, we can take $\left(i_{0}, i_{1}, i_{2}, i_{3}\right)=(1,2,3,4)$, i.e., $P_{\{1\}\{2,3,4\}}(\beta)=P_{\{1\}\{2,3,4\}}\left(\bigwedge^{2} Z(\beta)\right) \neq 0$. Thus $\bigwedge^{2} Z(\beta)=G(\omega)$ is indecomposable, proving 3 implies 2 .

Now we prove that 2 implies 1 . Suppose $\omega$ is indecomposable. We will prove the indecomposability of $\bigwedge^{p} X(\omega)$.

First consider the case where $\omega$ is the sum of two decomposable elements, $\omega=$ $v_{1} \wedge \cdots \wedge v_{p}+w_{1} \wedge \cdots \wedge w_{p}$. Setting $V=\left\langle v_{1}, \ldots, v_{p}\right\rangle_{Q}$ and $W=\left\langle w_{1}, \ldots, w_{p}\right\rangle_{Q}$ 
we have, as seen in the proof of Theorem 3.4, that $\operatorname{dim} V=\operatorname{dim} W=p$ and $\operatorname{dim} V \cap W \leq p-2$, so that $\operatorname{dim} V+W \geq p+2$. Using Lemma 5.2 with $V^{\prime}=V, W$ and $V+W$ respectively, we have $\operatorname{dim} X V=\operatorname{dim} X W=p$ and $\operatorname{dim}(X V+X W)=$ $\operatorname{dim}(X(V+W))=p+2$, so that $\operatorname{dim}(X V \cap X W)=p-2$. Hence $\bigwedge^{p} X(\omega)=$ $X v_{1} \wedge \cdots \wedge X v_{p}+X w_{1} \wedge \cdots \wedge X w_{p}$ is indecomposable.

Next we study the general case by induction on $n$. If $n=4$, then $\operatorname{det} X$ is a nonzero element of $R$, so that 2 implies 1 is obvious. Suppose the assertion holds for $n-1$. Let $\omega=\omega_{1} \wedge e_{n}+\omega_{2}$ with $\omega_{1} \in \bigwedge^{p-1} k^{n-1}$ and $\omega_{2} \in \bigwedge^{p} k^{n-1}$, where we regard $k^{n-1} \subset k^{n}$ as in the proof of Theorem 3.4. If both $\omega_{1}$ and $\omega_{2}$ are decomposable, it reduces to the case studied above.

Suppose $\omega_{1}$ is indecomposable. Let $X_{0}$ be the $(p+1) \times(n-1)$ matrix obtained from $X$ by removing the last row and column. Since $X e_{n}=\sum_{i=1}^{p+2} x_{i}^{n-1} e_{i}$, we have

$$
\begin{aligned}
\bigwedge^{p} X(\omega) & =\bigwedge^{p-1} X\left(\omega_{1}\right) \wedge X e_{n}+\bigwedge^{p} X\left(\omega_{2}\right) \\
& =(-1)^{p+n} x_{p+2}^{n-1} \bigwedge^{p-1} X_{0}\left(\omega_{1}\right) \wedge e_{p+2}+\text { lower degree terms in } x_{p+2} .
\end{aligned}
$$

By the induction hypothesis $\bigwedge^{p-1} X_{0}\left(\omega_{1}\right)$ is indecomposable, so the right-hand side of (14) is also indecomposable, as seen by expanding the Plücker relations $P_{A, B}\left(\bigwedge^{p} X(\omega)\right)$ with $A \cap B \ni p+2$ in powers of $x_{p+2}$ and taking the coefficients of $x_{p+2}^{2 n-2}$.

Suppose $\omega_{1}$ is decomposable and $\omega_{2}$ is not. Then $\bigwedge^{p-1} X\left(\omega_{1}\right)$ is decomposable, and by the induction hypothesis $\bigwedge^{p} X\left(\omega_{2}\right)$ is not. We prove by contradiction that $\bigwedge^{p} X(\omega)$ cannot be decomposable:

Let $\pi_{i_{1}, \ldots, i_{p-1}}^{\prime}$ (resp. $\left.\rho_{i_{1}, \ldots, i_{p}}\right)$ be the coordinates of $\bigwedge^{p-1} X\left(\omega_{1}\right)\left(\right.$ resp. $\left.\bigwedge^{p} X\left(\omega_{2}\right)\right)$. Thus $\bigwedge^{p} X\left(\omega_{1} \wedge e_{n}\right)=\bigwedge^{p-1} X\left(\omega_{1}\right) \wedge X e_{n}$ has the coordinates

$$
\pi_{i_{1}, \ldots, i_{p}}=\sum_{\nu=1}^{p}(-1)^{p-\nu} \pi_{i_{1}, \ldots, i_{p} \backslash i_{\nu}}^{\prime} x_{i_{\nu}}^{n-1} .
$$

By assumption, $\left\{\pi_{i_{1}, \ldots, i_{p}}\right\}$ satisfies all of the Plücker relations in $\mathcal{P}(p, p+2)$, and $\left\{\rho_{i_{1}, \ldots, i_{p}}\right\}$ does not satisfy some relation, say $P_{A, B}$, in $\mathcal{P}(p, p+2)$. Here, using the symmetry argument as we used in the proof of " 3 implies 2 " above, we may assume $A=\{1,5,6, \ldots, p+2\}$ and $B=\{2,3,4,5,6, \ldots, p+2\}$. Denoting the sequence of indices $5,6, \ldots, p+2$ by $\lambda$, and hence $\pi_{i j \lambda}=\pi_{i j 56 \ldots p+2}$, etc., we thus have

$$
\begin{aligned}
\pi_{12 \lambda} \pi_{34 \lambda}-\pi_{13 \lambda} \pi_{24 \lambda}+\pi_{14 \lambda} \pi_{23 \lambda} & =0, \\
\rho_{12 \lambda} \rho_{34 \lambda}-\rho_{13 \lambda} \rho_{24 \lambda}+\rho_{14 \lambda} \rho_{23 \lambda} & \neq 0 .
\end{aligned}
$$

If $\bigwedge^{p} X(\omega)$ is decomposable, then $\left\{\pi_{i_{1}, \ldots, i_{p}}+\rho_{i_{1}, \ldots, i_{p}}\right\}$, the coordinates of $\omega$, must satisfy all the relations in $\mathcal{P}(p, p+2)$ and so the above $P_{A, B}$ in particular; thus by using (16) we have

$$
\begin{aligned}
0= & \left(\pi_{12 \lambda}+\rho_{12 \lambda}\right)\left(\pi_{34 \lambda}+\rho_{34 \lambda}\right)-\left(\pi_{13 \lambda}+\rho_{13 \lambda}\right)\left(\pi_{24 \lambda}+\rho_{24 \lambda}\right) \\
& +\left(\pi_{14 \lambda}+\rho_{14 \lambda}\right)\left(\pi_{23 \lambda}+\rho_{23 \lambda}\right) \\
= & \left(\pi_{12 \lambda} \rho_{34 \lambda}-\pi_{13 \lambda} \rho_{24 \lambda}+\pi_{14 \lambda} \rho_{23 \lambda}\right)+\left(\rho_{12 \lambda} \pi_{34 \lambda}-\rho_{13 \lambda} \pi_{24 \lambda}+\rho_{14 \lambda} \pi_{23 \lambda}\right) \\
& +\left(\rho_{12 \lambda} \rho_{34 \lambda}-\rho_{13 \lambda} \rho_{24 \lambda}+\rho_{14 \lambda} \rho_{23 \lambda}\right) .
\end{aligned}
$$

By the definition of a linear map $X, \rho_{i j \lambda}$ are polynomials in $x_{r}, r=i, j, 5, \ldots, p+2$, with no $x_{r}$ having the $(n-1)$ st or higher power, and $\pi_{i j \lambda}$ are polynomials in the same set of $x_{r}$ with $x_{r}^{n-1}$ appearing only as the last factor in each term of (15). 
Moreover, for each $r \in\{1, \ldots, 4\}$, each term on the right-hand side of (18) is the product of a polynomial which depends on $x_{r}$ and another which does not. Thus comparing the coefficients of $x_{r}^{n-1}(r=2,3,4)$ on both sides of (18), we have

$$
\pi_{1 \lambda}^{\prime} \rho_{r^{\prime} r^{\prime \prime} \lambda}-\pi_{r^{\prime} \lambda}^{\prime} \rho_{1 r^{\prime \prime} \lambda}+\pi_{r^{\prime \prime} \lambda}^{\prime} \rho_{1 r^{\prime} \lambda}=0, \quad\left(r^{\prime}, r^{\prime \prime}\right)=(3,4),(4,2),(2,3) .
$$

After using these to eliminate $\rho_{23 \lambda}, \rho_{24 \lambda}$ and $\rho_{34 \lambda}$, the left-hand side of (17) becomes

$$
\rho_{12 \lambda} \frac{\pi_{3 \lambda}^{\prime} \rho_{14}-\pi_{4 \lambda}^{\prime} \rho_{13}}{\pi_{1 \lambda}^{\prime}}-\rho_{13 \lambda} \frac{\pi_{2 \lambda}^{\prime} \rho_{14 \lambda}-\pi_{4 \lambda}^{\prime} \rho_{12 \lambda}}{\pi_{1 \lambda}^{\prime}}+\rho_{14 \lambda} \frac{\pi_{2 \lambda}^{\prime} \rho_{13 \lambda}-\pi_{3 \lambda}^{\prime} \rho_{12 \lambda}}{\pi_{1 \lambda}^{\prime}}=0,
$$

which is a contradiction. Hence $\bigwedge^{p} X(\omega)$ is indecomposable, completing the proof of "2 implies 1".

\section{Concluding REMARKS}

We obtained a set $\mathcal{P}^{\prime}(p, n)$ of rank 6 quadratic forms on $\bigwedge^{p} k^{n}$, which is in general much smaller than the set of standard Plücker relations, and yet is capable of determining the decomposable elements in $\bigwedge^{p} k^{n}$. Every element of $\mathcal{P}^{\prime}(p, n)$ is obtained by pulling back the one nontrivial Plücker relation on $\bigwedge^{2} k^{4}$. This means:

1. The elements of $\mathcal{P}^{\prime}(p, n)$ define quadric hypersurfaces which are isomorphic to each other by a GCP map, and the Grassmannian is obtained as the intersection of those isomorphic quadrics. This may be of interest to geometers who have already noted and used the previously known fact that this was true when $p=2[13,14]$.

2. The Grassmannian $\operatorname{Gr}(p, n)$ is the intersection of the pullbacks of $\operatorname{Gr}(2,4)$ under all GCP maps from $\bigwedge^{p} k^{n}$ to $\bigwedge^{2} k^{4}$.

3. The 3 -term Plücker relation is in a sense "universal", providing an explanation for the special role played by the 3 -term relation in applications like soliton theory $[5,6]$. In this respect, upon suitable identification of variables, condition (4) in Theorem 5.3 can be viewed as a parameter-dependent Fay-Hirota type difference equation to characterize KP tau-functions $[12,17]$ in the case of rational solutions.

\section{ACKNOWLEDGMENTS}

The second and third authors worked on this paper as part of a summer undergraduate research experience at the College of Charleston, with the second author receiving financial support from the Department of Mathematics and the third author receiving financial support from the College's SURF program. We are grateful for assistance and advice from Malcolm Adams, Michael Gekhtman, Trygve Johnsen, Mitch Rothstein, Oleg Smirnov, Robert Varley and the referee.

\section{REFERENCES}

[1] S. Abeasis, Advances in Math. 36 (1980) 277-282 MR577305 (81m:14035)

[2] M.J. Bergvelt and A.P.E. ten Kroode, Pacific J. Math. 171 (1995), no. 1, 23-88 MR1362978 (97c:58061)

[3] N. Bourbaki, Algèbre. Chapitre III: Algèbre multilinéaire, Actualités Sci. Ind., no. 1044. Hermann et Cie., Paris, 1948

[4] C.C. Chevalley, The algebraic theory of spinors, Columbia University Press, New York, 1954 MR0060497 (15:678d)

[5] S. Duzhin, Decomposable skew-symmetric functions, Moscow Math J. 3 (2003) no. 3, 881-888 MR2078565 (2005f:15006)

[6] M. Gekhtman and A. Kasman, On KP generators and the geometry of the HBDE, Journal of Geometry and Physics 56 (2006) 282-309. MR2173898 (2006f:37097) 
[7] P. Griffiths and J. Harris, Principles of algebraic geometry, Reprint of the 1978 original, Wiley, New York, 1994 MR1288523 (95d:14001)

[8] W.V.D. Hodge and D. Pedoe, Methods of algebraic geometry. Vol. II, Reprint of the 1952 original, Cambridge Univ. Press, Cambridge, 1994 MR1288306 (95d:14002b)

[9] S.L. Kleiman and D. Laksov, American Mathematical Monthly 79 (1972) 1061-1082 MR0323796 (48:2152)

[10] I. Krichever, "Characterizing Jacobians via trisecants of the Kummer Variety", math.AG/0605625

[11] I.G. Macdonald, Symmetric functions and Hall polynomials, 2nd Ed., Oxford University Press, Oxford, 1995 MR1354144 (96h:05207)

[12] T. Miwa, Proc. Japan Acad. Ser. A Math. Sci. 58 (1982) no. 1, 9-12 MR649054 (83f:58042)

[13] S. Mukai, in Algebraic geometry and related topics (Inchon, 1992), 19-40, Internat. Press, Cambridge, MA, 1993 MR1285374 (95i:14032)

[14] K. Ranestad and F.-O. Schreyer, Varieties of sums of powers, J. Reine Angew. Math. 525 (2000), 147-181 MR1780430 (2001m:14009)

[15] M. Sato and Y. Sato, in H. Fujita, P.D. Lax, G. Strang (eds) Nonlinear partial differential equations in applied science (Tokyo, 1982), 259-271, North-Holland, Amsterdam, 1983 MR730231 (84m:35002)

[16] R. Stanley, Enumerative combinatorics, vol. 2, Cambridge Univ. Press, Cambridge, UK, 1999 MR1676282 (2000k:05026)

[17] K. Takasaki and T. Takebe, Rev. Math. Phys. 7 (1995) 743-808 MR1346289 (97f:58073)

[18] R. Westwick, Linear and Multilinear Algebra 2 (1974/75), 257-268 MR0429961 (55:2969)

Department of Mathematics, College of Charleston, 66 George Street, Charleston, South Carolina 29424

E-mail address: kasman@cofc.edu

Department of Mathematics, College of Charleston, 66 George Street, Charleston, South CARolina 29424

E-mail address: kepedings@edisto.cofc.edu

Department of Mathematics, College of Charleston, 66 George Street, Charleston, South Carolina 29424

E-mail address: amreiszl@edisto.cofc.edu

Department of Mathematics, Kyoto University, Kyoto, 606-8502, Japan 